\title{
Authentication of Oviductus Ranae and Its Original Animals Using Molecular Marker
}

\author{
Yang Xuegan, Wang Yiquan, ${ }^{*}$ Zhou KaIYA, and Liu Zhongquan \\ Institute of Genetic Resources, Nanjing Normal University; Nanjing 210097, China. \\ Received October 22, 2001; accepted April 12, 2002
}

\begin{abstract}
Two pairs of diagnostic primers, IHm01-L/IHm01-H and IHm02-L/IHm02-H, for distinguishing the Chinese crude drug Oviductus Ranae from its substitutes were designed based on sequences of Cyt $b$ gene fragment of the original animals of the drug and substitutes. Total DNAs were extracted from crude drugs purchased from five drugstores in different regions, as well as from original animals of the drug, Rana chensinensis, and seven species of related ranid species. Diagnostic polymerase chain reactions (PCRs) were performed using the two pairs of primers with the total DNAs of the original animals as a template. The result showed that a $240 \mathrm{bp}$ DNA segment was clearly amplified from all templates of Rana chensinensis using primers IHm01-L and IHm01-H, whereas no DNA band appeared from other templates. While using primers IHm02-L and IHm02-H, we got a clear $140 \mathrm{bp}$ DNA band from all the templates of $R$. huanrenensis and 3 oviducts of the same species, no PCR product was observed from the other samples. A set of PCR reactions was employed to identify crude drugs from the five drugstores using the two pairs of primers together with HsmL1 and HsmH1 reported in our previous study. The results show that only $20 \%$ of the Oviductus Ranae currently sold in markets are qualified products and the rest are not.
\end{abstract}

Key words Oviductus Ranae; allele specific primer; diagnostic polymerase chain reaction (PCR)

Oviductus Ranae, a valuable Chinese crude drug, is recorded in Pharmacopoeia of the People's Republic of China (2000 edition) as a dried oviduct of the female Chinese brown frog, Rana chensinensis, distributing mainly in northeastern China. In the application of Chinese medicine, Oviductus Ranae is used to replenish the kidney essence, to nourish the yin, and to moisten the lung. ${ }^{1)}$ Unfortunately, substitutes or adulterants of Oviductus Ranae are found on the Chinese medicinal market, and the most common ones are made of oviducts of Bufo gargarizans gargarizans, Pelophylax nigromaculata, or P. plancyi plancyi. ${ }^{2}$ ) Swelling capacity is the criterion recommended in the Chinese Pharmacopoeia to identify the genuine product. But it varies greatly with water temperature, soaking duration, and even among individuals, and thus is unsatisfactory in most cases. ${ }^{3)}$ It is also very difficult to distinguish the actual product from its substitutes/adulterants by morphological or chemical characteristics. ${ }^{3)}$ In our previous report, we designed a pair of allele specific primers to identify the genuine product of Oviductus Ranae based on DNA sequence of $12 \mathrm{~S}$ rRNA gene. ${ }^{4)}$ However, this gene is relatively conservative with less variation among species of frogs. Although we can discriminate three brown frogs: $R$. chensinensis, $R$. amurensis and $R$. huanrenensis from other frogs and toads by allele specific polymerase chain reaction (PCR) using this primer pair, we are still unable to distinguish $R$. chensinensis from the other two kinds of brown frogs. The purpose of the present study was to design more sensitive primer pairs based on mitochondrial Cyt $b$ sequences to identify each of the three brown frogs.

\section{MATERIALS AND METHODS}

Samples Samples of Oviductus Ranae were purchased respectively from 5 drugstores in China: Sichuan Derentang Traditional Chinese Medicinal Materials Co. (Sichuan), Dalian Traditional Chinese Medicinal Materials Co. (Dalian), Beijing Tongrentang Traditional Chinese Medicinal
Materials Co. (Beijing), Jiangsu Traditional Chinese Medicinal Materials Co. (Jiangsu) and Nanjing Cunxintai Traditional Chinese Medicinal Materials Shop (Nanjing). Producing area of these samples was all labeled as northeastern China by the vendors. We selected 10 samples randomly from each purchasing panel and 3 oviduct samples taken from the original animals of $R$. huanrenensis for DNA extraction and PCR identification.

For DNA sequencing and testing primers designed for authentication of the drugs, a total of 9 species of the original animal including 24 individuals covering all reported substitute species were sampled and their taxonomic assignment follows $\mathrm{Fei}^{5)}$ (Table 1).

DNA Extraction and Amplification The procedure of DNA extraction from both crude drugs and original animals was the same as that described in a previous report. ${ }^{4)}$

Based on the analysis of DNA sequence data of human, ${ }^{6}$ Xenopus laevis ${ }^{7)}$ and some Ranid frogs, ${ }^{8-11)}$ a pair of universal primers L14847 (5'-ACATCTCAGCATGATGAAACTTCGG-3') and H15230 (5'-TACTGAGAAGCCCCCTCAAATTCATTG-3') was designed for amplifying Cyt $b$ gene fragment from all frogs mentioned above in the present study. A total of $30 \mu \mathrm{l}$ of PCR reaction mixture was composed of $10 \times$ buffer $3 \mu \mathrm{l}, 25 \mathrm{mmol} / 1 \mathrm{Mg}^{2+} 2 \mu \mathrm{l}, 2 \mathrm{mmol} / 1 \mathrm{dNTPs} 2 \mu \mathrm{l}$, $10 \mathrm{pmol} / \mu \mathrm{l}$ of each universal primer $1 \mu \mathrm{l}, 1$ unit of Taq DNA polymerase (Promega), $10 \mathrm{mg} / \mathrm{ml}$ BSA $0.2 \mu \mathrm{l}$, and template DNA $10-100 \mathrm{ng}$. Amplification was carried out in a GeneAmp PCR System 2400 (Perkin Elmer) or PTC-200 thermocycler (MJ Research). Samples were denatured at $95^{\circ} \mathrm{C}$ for $5 \mathrm{~min}$ and subjected to 30 cycles of $40 \mathrm{~s}$ at $95^{\circ} \mathrm{C}, 40 \mathrm{~s}$ at $52^{\circ} \mathrm{C}, 90 \mathrm{~s}$ at $72^{\circ} \mathrm{C}$, and a final extension of $7 \mathrm{~min}$ at $72^{\circ} \mathrm{C}$.

Sequencing of PCR Product PCR products were purified using a DNA purification kit (Shanghai Watson Bioengineering Inc.) according to the manufacturer's instruction, and then sequenced with BigDye ${ }^{\mathrm{TM}}$ on an ABI PRISM ${ }^{\mathrm{TM}} 310$ Genetic Analyzer (Perkin Elmer). Nonspecific amplicons existing in some reactions usually generated a strong back- 
Table 1. Samples of Original Animal Used in Present Study

\begin{tabular}{|c|c|c|c|}
\hline Species $\left.^{a}\right)$ & Code & Number & Source \\
\hline Rana chensinensis (M) & Rchem & 3 & Mudanjiang, Heilongjiang \\
\hline Rana chensinensis (S) & Rches & 5 & Shenyang, Liaoning \\
\hline Rana chensinensis (Y) & Rchey & 1 & Yuzhong, Gansu \\
\hline R. amurensis & Ramum & 5 & Mudanjiang, Heilongjiang \\
\hline R. huanrenensis & Rhuah & 3 & Huanren, Liaoning \\
\hline R. zhenhaiensis & Rzhez & 1 & Zhenhai, Zhejiang \\
\hline R. chaochiaoensis & Rcham & 1 & Muli, Sichuan \\
\hline R. omeimontis & Romee & 1 & Emei, Sichuan \\
\hline Pelophylax nigromaculata & Pnigh & 1 & Huoqiu, Anhui \\
\hline P.p.plancyi & Pplaw & 1 & Wuhu, Anhui \\
\hline Bufo g. gargarizans & Bufo & 2 & Nanjing, Jiangsu \\
\hline
\end{tabular}

a) The species of original animals are named following ref. 5 .
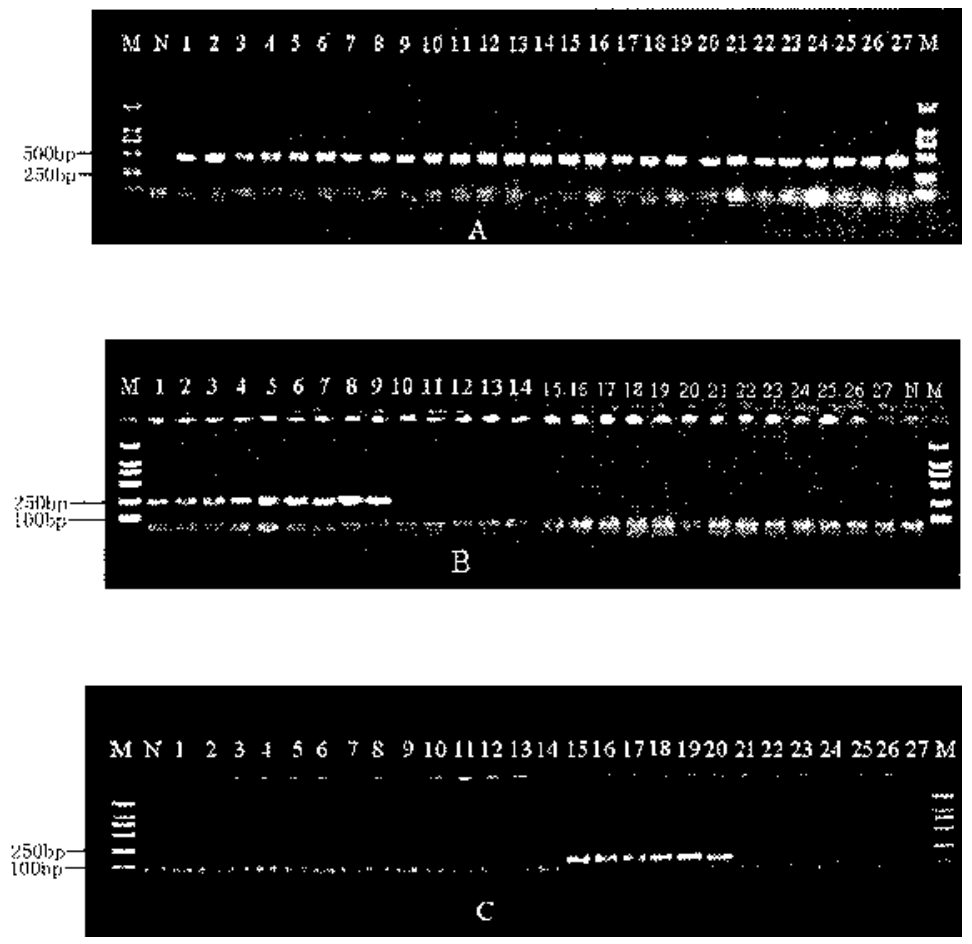

Fig. 1. Agarose Gel Electrophoresis of PCR Identification of Original Animals

A: Positive control with universal primers. B: Allele specific PCR with IHm01-L and IHm01-H. C: Allele specific PCR with IHm02-L and IHm02-H. 1-9: Rana chensinensis, 10-14: R. amurensis, 15-17: R. huanrenensis, 18-20: 3 oviducts of $R$. huanrenensis, 21: R. zhenhaiensis, 22: $R$. omeimontis, 23: R. chaochiaoensis, 24: Pelophylax nigromaculata, 25: P. p. plancyi, 26-27: Bufo g. gargarizans, M: DNA marker, DL2000 (TaKaRa), N: negative control without DNA template.

ground during DNA sequencing. To solve this problem, a strategy of TA cloning sequencing was employed in our experiments. The vector PMD18-T was purchased from TaKaRa Co. Recombined plasmid was extracted with a QIAprep spin miniprep kit (QIAGEN) and sequenced as described above.

Diagnostic PCR Based on DNA sequence data of Cyt $b$ gene fragment obtained in the present research and cited from published papers, we designed two pairs of allele specific primers, IHm01-L (5'-TTTCATCTATCGCCCATATCTGCCGG-3') and IHm01-H (5'-CAGAAGGATATTTGGCCTCATGGTAAC-3'), IHm02-L (5'-ACTTCTCTGGCATTCTCATCTATCGCA-3') and IHm02-H (5'-CCATAATAAAGACCTCGCCCAATATGA-3'), for the identification of Oviductus Ranae. The first pair was specialized to identify $R$. chensinensis, and could be used to specifically amplify a 240 bp DNA segment from a template of this species only.
The second pair was specialized to label $R$. huanrenensis, and a 140 bp DNA band would be generated only from $R$. huanrenensis DNA in the amplification. Diagnostic PCRs were performed under several annealing temperatures to estimate a suitable range of temperature variation. Positive controls were also performed for the same set of templates using universal primers L14847 and $\mathrm{H} 15230$ annealing at $52^{\circ} \mathrm{C}$. PCR products were tested by electrophoresis in $1.5 \%$ agarose gel stained with ethidium bromide.

\section{RESULTS}

About 360 bp Cyt $b$ gene fragment was amplified from 16 samples, $R$. chensisnensis (5 individuals), $R$. amurensis (3 individuals), $R$. huanrenensis (3 individuals), $R$. zhenhaiensis, $R$. chaochiaoensis, $R$. omeimontis, Pelophylax nigromaculata, and P. p. plancyi, and then sequenced directly or via 
cloning. The sequences have been submitted to GenBank with accession numbers of AF274914-AF274924 and AF274926 - 274930. Corresponding Cyt $b$ sequence data of Bufo g. gargarizans was cited from GenBank (accession number AF171195). After alignment of these sequences, genetic distance was estimated as $0.3-6.4 \%$ among 5 samples of $R$. chensinensis, $11.4-19.3 \%$ between $R$. chensinensis and the other two species of brown frogs from northeastern China, 16.7-19.0\% between R. chensinensis and Pelophylax nigromaculata or P. p. plancyi, and 58.6-61.6\% between $R$. chensinensis and Bufo g. gargarizans (Table 3).

Before diagnostic PCR for authentication of crude drugs, we employed the universal primer pair and the two allele specific primer pairs to amplify DNA templates of the 27 original animals (including 3 oviducts of $R$. huanrenensis) to verify the primers. The result of amplification showed that a 240 bp DNA segment was clearly amplified from 9 templates of $R$. chensinensis annealing at $60^{\circ} \mathrm{C}$ using primers IHm01-L and $\mathrm{IHm} 01-\mathrm{H}$, whereas no DNA band appeared in amplification with other templates under the same reaction conditions. When annealing temperature rose to $70^{\circ} \mathrm{C}$, the DNA segment was still amplified well from $R$. chensinensis templates. While using primers IHm02-L and IHm02-H with an annealing temperature at $55^{\circ} \mathrm{C}$, we got a clear $140 \mathrm{bp}$ DNA band from each reaction containing a template from $R$. huanrenensis and 3 oviducts of the same species, but no PCR product was observed in the amplifications of the other samples. The results were repeated well. Positive controls for all DNA templates produced a DNA fragment about $400 \mathrm{bp}$ in size, indicating that all templates are qualified for PCR (Fig. 1).

In the process of crude drug identification, about a $400 \mathrm{bp}$ DNA fragment was amplified from all 50 templates randomly sampled from 5 purchasing panels using universal primers with annealing at $52{ }^{\circ} \mathrm{C}$, indicating the DNA from
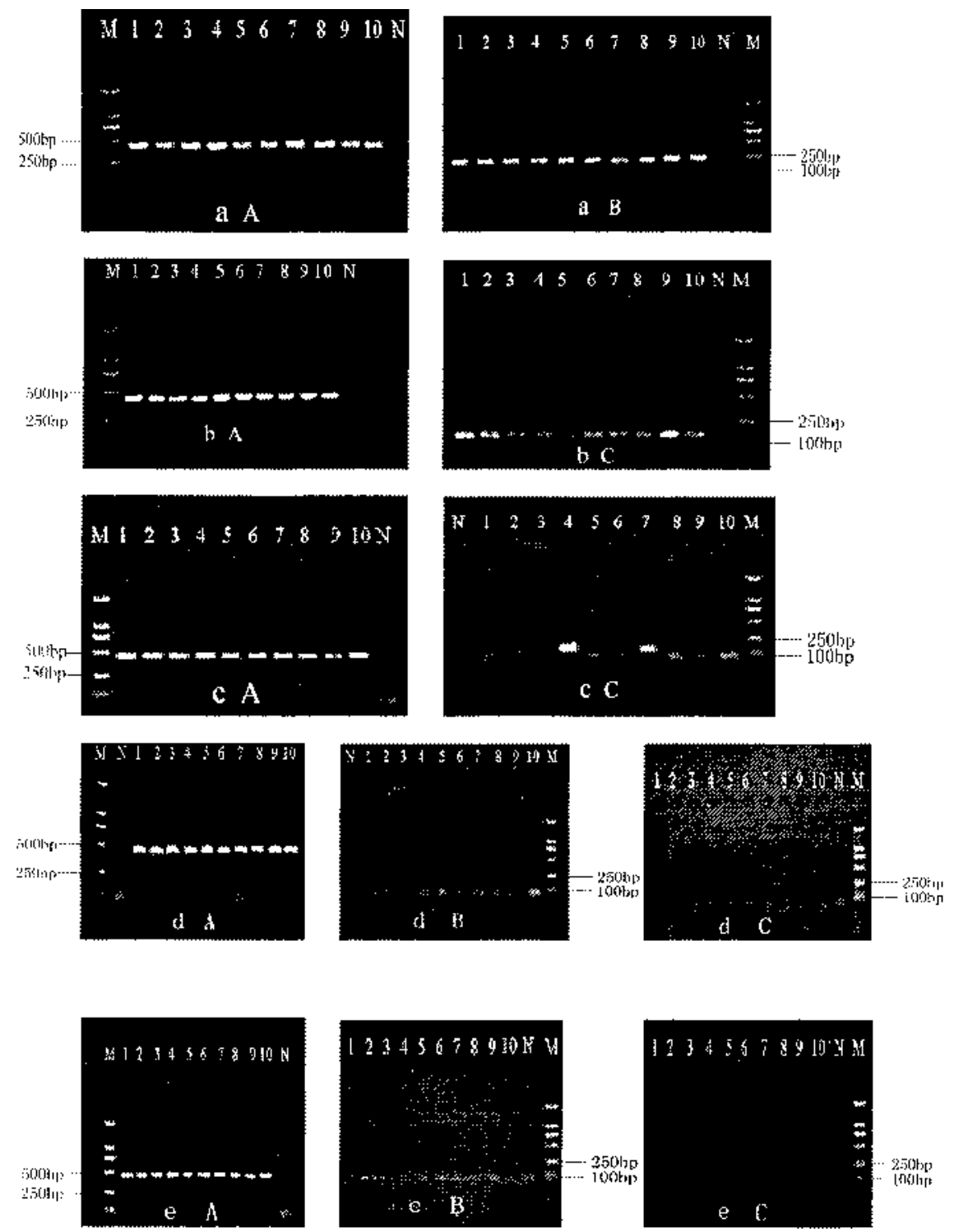

Fig. 2. Agarose Gel Electrophoresis of PCR Identification of Crude Drugs

The lower cases at the bottom of each picture indicate samples originated from different regions. a: Sichuan Denrentang Tradition Chinese Medicinal Material Co., b: Dalian Tradition Chinese Medicinal Material Co., c: Beijing Tongrentang Tradition Chinese Medicinal Material Co., d: Jiangsu Tradition Chinese Medicinal Material Co., e: Nanjing Cunxintai Tradition Chinese Medicinal Material Shop, 1 10: number of samples, M: DNA marker, DL2000 (TaKaRa), N: negative control without DNA template. The capital letters at the bottom of pictures indicate PCRs performed with different primer pairs. A: Positive control with universal primers. B: Allele specific PCR with IHm01-L and IHm01-H. C: Allele specific PCR with IHm02-L and IHm02-H. 
Table 2. PCR Identification Results of 10 Samples from Each Purchasing Panel Using Diagnostic Primers

\begin{tabular}{|c|c|c|c|c|}
\hline \multirow{2}{*}{ Purchasing panel } & \multicolumn{3}{|c|}{ Primer pairs } & \multirow[b]{2}{*}{ IHm02-L/IHm02-H } \\
\hline & L14847/H15230 & HsmL1/HsmH1 & IHm01-L/IHm01-H & \\
\hline Sichuan & ++++++++++ & ++++++++++ & ++++++++++ & --------- \\
\hline Dalian & ++++++++++ & ++++++++++ & --------- & ++++++++++ \\
\hline Jiangsu & ++++++++++ & ++++++++++ & --------- & --------- \\
\hline Beijing & ++++++++++ & ---+--+--- & --------- & ---+--+--- \\
\hline Nanjing & ++++++++++ & +-------+ & --------- & --------- \\
\hline
\end{tabular}

Note: "+" and "-" denote positive and negative amplifications, respectively. Positive amplifications using L14847/H15230 show all samples are qualified for PCR reaction. Samples generating positive amplifications with primer $\mathrm{IHm} 01-\mathrm{L} / \mathrm{IHm} 01-\mathrm{H}$ are $R$. chensinensis, and those generating positive amplifications with $\mathrm{IHm} 02-\mathrm{L} / \mathrm{IHm} 02-\mathrm{H}$ are $R$. huanrenensis. Samples generating positive amplifications with HsmL1/HsmH1 but negative amplifications with IHm01-L/IHm01-H or IHm02-L/IHm02-H are R. amurensis. The species names of samples generating negative amplifications with all primer pairs except L14847/H15230 are uncertain but not brown frogs.

Table 3. Numbers of Transitions/Transversions (Upper Triangle) and the Percentage of Sequence Diversity (Lower Triangle) for Cyt $b$ Gene of 9 Species of Original Animal Examined with Gaps Deleted in Each Pairwise Comparison

\begin{tabular}{|c|c|c|c|c|c|c|c|c|c|c|c|c|c|c|c|c|c|c|}
\hline & Code $^{a)}$ & 1 & 2 & 3 & 4 & 5 & 6 & 7 & 8 & 9 & 10 & 11 & 12 & 13 & 14 & 15 & 16 & 17 \\
\hline 1 & Rchem 1 & & 0 & 0 & $1 / 5$ & $17 / 3$ & $36 / 10$ & $37 / 7$ & $39 / 9$ & $52 / 15$ & $52 / 15$ & $52 / 16$ & $42 / 17$ & $40 / 12$ & $43 / 18$ & $38 / 25$ & $39 / 25$ & $101 / 118$ \\
\hline 2 & Rchem 2 & 0.3 & & 0 & $1 / 4$ & $17 / 2$ & $36 / 9$ & $39 / 8$ & $39 / 8$ & $52 / 14$ & $52 / 14$ & $52 / 15$ & $42 / 16$ & $40 / 11$ & $43 / 17$ & $38 / 24$ & $39 / 24$ & $103 / 117$ \\
\hline 3 & Rches 1 & 0.6 & 0.3 & & $1 / 5$ & $17 / 3$ & $36 / 10$ & $39 / 9$ & $39 / 9$ & $52 / 15$ & $52 / 15$ & $52 / 16$ & $42 / 17$ & $40 / 12$ & $43 / 18$ & $38 / 25$ & $39 / 25$ & $102 / 118$ \\
\hline 4 & Rches 2 & 1.8 & 1.5 & 1.8 & & $15 / 4$ & $30 / 11$ & $36 / 10$ & $35 / 10$ & $48 / 16$ & $48 / 16$ & $48 / 17$ & $41 / 18$ & $38 / 13$ & $42 / 19$ & $38 / 25$ & $39 / 25$ & $91 / 113$ \\
\hline 5 & Rchey1 & 6.4 & 6.1 & 6.4 & 6.3 & & $33 / 7$ & $32 / 5$ & $31 / 5$ & $44 / 10$ & $44 / 10$ & $43 / 11$ & $37 / 11$ & $31 / 7$ & $38 / 13$ & $33 / 20$ & $30 / 22$ & $82 / 99$ \\
\hline 6 & Ramum 1 & 13.8 & 13.5 & 13.8 & 11.4 & 12.8 & & $3 / 4$ & $2 / 4$ & $48 / 11$ & $48 / 11$ & $48 / 12$ & $39 / 14$ & $31 / 10$ & $44 / 12$ & $41 / 21$ & $39 / 23$ & $95 / 109$ \\
\hline 7 & Ramum 2 & 12.8 & 13.1 & 13.3 & 13.7 & 11.9 & 2.1 & & $1 / 2$ & $49 / 10$ & $49 / 10$ & $49 / 11$ & $42 / 12$ & $35 / 9$ & $45 / 11$ & $42 / 20$ & $40 / 22$ & $106 / 115$ \\
\hline 8 & Ramum 3 & 13.3 & 13.1 & 13.3 & 13.4 & 11.5 & 1.8 & 0.8 & & $49 / 10$ & $49 / 10$ & $49 / 11$ & $42 / 10$ & $36 / 9$ & $47 / 9$ & $44 / 18$ & $42 / 20$ & $109 / 113$ \\
\hline 9 & Rhuah 1 & 18.6 & 18.3 & 18.6 & 19.0 & 17.3 & 17.7 & 16.4 & 16.4 & & 1 & 0 & $41 / 18$ & $44 / 13$ & $26 / 13$ & $35 / 22$ & $34 / 24$ & $104 / 117$ \\
\hline 10 & Rhuah 2 & 18.6 & 18.3 & 18.6 & 19.0 & 17.3 & 17.7 & 16.4 & 16.4 & 0.3 & & 1 & $41 / 18$ & $44 / 13$ & $26 / 13$ & $34 / 22$ & $33 / 24$ & $106 / 117$ \\
\hline 11 & Rhuah 3 & 18.9 & 18.6 & 18.9 & 19.3 & 17.3 & 18.0 & 16.7 & 16.7 & 0.3 & 0.6 & & $41 / 19$ & $43 / 14$ & $26 / 14$ & $48 / 20$ & $52 / 15$ & $104 / 118$ \\
\hline 12 & Rzhez 1 & 16.4 & 16.1 & 16.4 & 17.6 & 15.4 & 15.9 & 15.0 & 14.4 & 16.4 & 16.4 & 16.7 & & $33 / 7$ & $33 / 9$ & $36 / 24$ & $37 / 26$ & $110 / 113$ \\
\hline 13 & Rcham 1 & 14.7 & 14.4 & 14.7 & 15.2 & 12.2 & 12.3 & 12.5 & 12.7 & 16.1 & 16.1 & 16.1 & 11.3 & & $38 / 8$ & $37 / 23$ & $37 / 25$ & $108 / 112$ \\
\hline 14 & Romee 1 & 16.9 & 16.7 & 16.9 & 18.2 & 16.3 & 16.8 & 15.6 & 15.6 & 10.8 & 10.8 & 11.1 & 11.7 & 13.0 & & $33 / 21$ & $34 / 23$ & $103 / 118$ \\
\hline 15 & Pnigh 1 & 17.5 & 17.2 & 17.5 & 18.8 & 17.0 & 18.6 & 17.2 & 17.2 & 15.8 & 15.6 & 15.8 & 16.7 & 17.0 & 15.0 & & $3 / 2$ & $102 / 115$ \\
\hline 16 & Pplaw 1 & 17.8 & 17.5 & 17.8 & 19.0 & 16.7 & 18.6 & 17.2 & 17.2 & 16.1 & 15.8 & 16.1 & 17.5 & 17.6 & 15.8 & 1.4 & & $99 / 117$ \\
\hline 17 & Bufo & 61.5 & 61.6 & 61.6 & 60.1 & 58.6 & 61.6 & 61.9 & 62.2 & 61.9 & 62.5 & 62.2 & 62.5 & 62.3 & 61.9 & 60.8 & 60.5 & \\
\hline
\end{tabular}

a) See Table 1 for explanation of the code.

these crude drugs were good enough for PCR (Fig. 2, Table 2). Diagnostic PCR for these samples with primers HsmL1/HsmH1 marked on 12S rRNA gene showed positive results in most cases, and these positive amplifications implied the templates originated from one of the three brown frogs mentioned above. ${ }^{4}$

For more accurate identification, further allele specific PCRs were carried out using IHm01-L and IHm01-H annealing at $60^{\circ} \mathrm{C}$, IHm02-L and $\mathrm{IHm} 02-\mathrm{H}$ annealing at $55^{\circ} \mathrm{C}$. In the former reaction, a $240 \mathrm{bp}$ DNA fragment was amplified only from 10 Sichuan samples, which indicated the original animal of these crude drugs was $R$. chensinensis. In the latter, a 140 bp DNA fragment was amplified from 10 Dalian samples and 2 Beijing samples (Fig. 2, Table 2). The results suggested these crude drugs were originated from $R$. huanrenensis.

\section{DISCUSSION}

For the authentication of Oviductus Ranae, allele specific primers HsmL1/HsmH1 located on 12S rRNA gene could be used to distinguish the crude drugs made of $R$. chensinensis, $R$. amurensis and $R$. huanrenensis from those made of other ranid species. But the primer pair was unable to separate the crude drugs made of the three brown frogs. ${ }^{4)}$ Cyt $b$ gene with a higher variation rate has been proved a good molecular marker for close affinitive species in the authentication of some animal crude drugs. ${ }^{12,13)}$ For more perfect identification, the gene of Cyt $b$ was selected as a DNA molecular marker to single out each brown frog species. After aligning and comparing a total of 24 sequences of this gene, including 8 sequences obtained from published data, we designed two pairs of specified primers for identification of $R$. chensinensis and $R$. huanrenensis, respectively. Because of the limitation of available data, no proper position on the current segment was found for designing primers that would be specialized only for $R$. amurensis. However, if we use these two pairs of primers together with HsmL1/HsmH1 reported in our previous study to perform a set of PCR reactions, the name of the above species could be figured out easily (Table 2).

Ten samples were randomly selected from each purchasing panel for PCR identification using the above allele specific primers. The results showed that the 10 samples from Sichuan originated from $R$. chensinensis, and the 10 samples from Dalian and 2 samples from Beijing originated from $R$. huanrenensis. Those from Jiangsu and Nanjing as well as 8 samples from Beijing were rejected by both IHm01$\mathrm{L} / \mathrm{IHm} 01-\mathrm{H}$ and $\mathrm{IHm} 02-\mathrm{L} / \mathrm{IHm} 02-\mathrm{H}$ amplification. In these rejected samples, 10 from Jiangsu and 2 from Nanjing were accepted by $\mathrm{HsmL} 1 / \mathrm{HsmH} 1$ amplification. Therefore, we presumed that they originated from $R$. amurensis, and from them we randomly selected two samples for further sequence 
analysis. Only one or two varied sites were found when comparing the $240 \mathrm{bp}$ DNA segment of Cyt $b$ gene with that of $R$. amurensis. Sequence variation rates among the three individuals are 0.4 to $0.8 \%$, which indicates they are conspecific.

The results in Table 2 also show that only 10 samples of one purchasing panel originated from $R$. chensinensis, 12 samples were from $R$. huanrenensis, another 12 samples were from $R$. amurensis and the rest from unknown ranid frogs but not brown frogs. In other words, only twenty percentage of Oviductus Ranae purchased from the market are genuine products. Traditionally, dried oviduct of $R$. huanrenensis or $R$. amurensis is also served as Oviductus Ranae by local people in northeastern China. However, no research on their pharmacological effect has been published and these substitutes have not yet been authorized officially by Chinese Pharmacopoeia.

Acknowledgements The authors would like to express their thanks to Dr. Jiang Jianping for offering and identifying some animal samples. The manuscript has benefitted greatly from the constructive comments and helpful suggestions of anonymous referees. This work is supported by NSFC (No. 39870913), the foundation for Univ. Key Teacher by the Ministry of Education of China, grants from the Jiangsu Educational Committee (No. 98KJB360010) and the 333 Project of Jiangsu Province, China.

\section{REFERENCES}

1) The Pharmacopoeia Commission of PRC (ed.), "Pharmacopoeia of the People's Republic of China (English Edition 2000)," The People's Medical Publishing House, 142.

2) Zhao W. Y., Hou Z. R., Sun G. C., J. Shenyang Pharma. Univ., 13, 68-71 (1996).

3) He B. Z., Xie L. S., Bull. Chin. Mater. Med., 12, 583-585 (1987).

4) Yang X. G., Wang Y. Q., Zhou K. Y., Liu Z. Q., Zhao W. G., Chin. J. Appl. Environ. Biol., 6, 166-170 (2000).

5) Fei L. (ed.), "Atlas of Amphibians of China," Henan Publishing House of Science and Technology, 1999.

6) Anderson S., Bankier A. T., Barrell B. G., de Bruijin M. H. L., Coulson A. R., Drouin J., Eperon I. C., Nierlich D. P., Roe B. A., Sanger F., Schreier P. H., Smith A. J. H., Staden R., Young I. G., Nature (London), 290, 457-465 (1981).

7) Roe B. A., Ma D. P., Wilson R. K., Wong J. F. H., J. Bio. Chem., 260 , 9759-9774 (1985).

8) Tanaka T., Matsui M., Takenaka O., Zoological Science, 11, 753-757 (1994).

9) Tanaka T., Matsui M., Takenaka O., Bioch. Syst. Ecol., 24, 299-307 (1996).

10) Tanaka-Ueno T., Matsui M., Chen S. L., Takenaka O., Ota H., Zoological Science, 15, 283-288 (1998).

11) Tanaka-Ueno T., Matsui M., Sato T., Takenaka S., Takenaka O., Zoological Science, 15, 289-294 (1998).

12) Wang Y. Q., Zhou K. Y., Xu L. S., Dong T. T. X., Tsim K. W. K., Biol. Pharm. Bull., 23: 585-588 (2000).

13) Wang Y. Q., Zhou K. Y., Xu L. S., Xu G. J., Acta Pharm. Sini., 33, 941—947 (1998). 